\title{
Integrated Metaheuristic Differential Evolution Optimization Algorithm and Pseudo Static Analysis of Concrete Gravity Dam
}

\author{
Taher Memarian ${ }^{a}$, Yaser Shahbazi ${ }^{\mathrm{b} *}$ \\ ${ }^{a}$ M.S. of Hydraulic Structures, Islamic Azad University Bandar Abbas Branch, Bandar Abbas, Iran. \\ ${ }^{b}$ Assistant Professor, Architecture and Urbanism Department, Tabriz Islamic Art University, Tabriz, Iran.
}

Received 07 February 2017; Accepted 28 August 2017

\begin{abstract}
A differential evolution-algorithm-based optimum design method is presented for concrete gravity dams under stability, principal stress, and sliding constraints. A gravity dam is a large scale hydraulic structure providing its stability based on weight of concrete volume. Hence, optimization of dam cross-section leads to an economic and low cost structure. For this aim, a general dam section is reconstructed with seven proper horizontal and vertical geometric parameters which take into account all possible cross section shapes. Weight of dam is considered as goal function and the optimization problem of geometric parameters is solved using DE algorithm. The DE algorithm written as a MATLAB code are applied to Four benchmark gravity dams including Middle Fork, Richard, Pine Flat, and Friant. The comparison of DE optimum solutions with real dimension of dams and another optimization method in literature shows the performance of the DE algorithm. In mentioned benchmark dams, there are $26.82 \%, 30.11 \%, 25.31 \%$, and $20.93 \%$ of weight reduction Compared to real values, respectively. Also, optimization results of DE algorithm are compared with literatures. The comparison shows $3.55 \%$, $5.1 \%, 19.13 \%$ and $12.14 \%$ reduction of weight compared to GA and PSOD algorithms, respectively.
\end{abstract}

Keywords: Gravity Dam; Optimization; Metaheuristic Algorithms; Differential Evolution.

\section{Introduction}

Optimum design can be defined as the best acceptable design, based on a predetermined criterion of qualitative merit. The importance of designing structures with minimum weight was first considered by the aerospace industries in which the design of the plane is controlled majorly by weight than cost. In other industries related to civil engineering, cost may have the first importance even though the weight of system impresses its cost and function. So, optimization issue plays a great role in the ideal world. Existing optimization methods can be generally classified as analytical and numerical approaches. In Analytical approaches, the optimal point of continuous and differentiable goal function is determined using differential calculus. Due to nonlinear, discontinuous and non-differentiable goal function of real problems with various design constraints, analytical optimization may not be effective in civil engineering. Whilst the numerical approaches, especially metaheuristic algorithms, show high acceptability for solving complex and nonlinear design problems in civil engineering.

Various old and famous metaheuristic algorithms such as genetic algorithm [1-4], genetic programming [5-7], ant colony optimization [8-10], bat algorithm [11-13], and particle swarm optimization [14-16] are introduced in literature of civil engineering studies. Recently, new metaheuristic algorithms are developed by researchers for example Big bang big crunch algorithm by [17], Charged System Search by [18], ray optimization by [19], and flower pollination algorithm by [20]. The metaheuristic algorithms in civil engineering problems and their recent applications are presented by [21].

\footnotetext{
* Corresponding author: y.shahbazi@tabriziau.ac.ir

$>$ This is an open access article under the CC-BY license (https://creativecommons.org/licenses/by/4.0/).

(C) Authors retain all copyrights.
} 
In concrete gravity Dams, weight and strength of the concrete maintain stability and equilibrium against external loads. Due to high volume of consumer concrete, a shape optimization design leads to a remarkably reduction of construction cost. Hence, researchers have shown an Impressive interest in employing metaheuristic approaches to obtain optimal shape of gravity dams. Genetic algorithm optimum design of gravity dam is presented in [22]. In this research, optimal top width of gravity dam is optimized with genetic algorithm under stability and stress constrains. Also, [23] were developed genetic algorithm based model for optimal design of a gravity dam under seismic excitation with reservoirdam foundation interactions. The particle swarm algorithm coupled with ANSYS software is used to carry out optimum design of gravity dam [24]. Three recently developed metaheuristic algorithms including charged system search (CSS), colliding bodies optimization (CBO) and its enhanced edition (ECBO) are utilized for optimizing the gravity dam by [25]. The shape optimization problem of gravity dam is considered under stability and principal stress constraints. GA and PSOD optimization algorithm are utilized to minimize the cross section of gravity dam by [26]. Dam-waterfoundation interaction and nonlinear effects due to earthquake of gravity dams are considered in optimization problem [27].

This paper presents differential evolution based optimization model of each possible cross section of gravity dams. The DE algorithm, a smart metaheuristic algorithms based on probability law with local search ability, is introduced by [28]. A general cross section of gravity dam is constructed using various unknown geometric parameters. The horizontal and vertical forces actin on gravity dam and corresponding moments about toe which may affect the pseudo static analysis of gravity dam are written based on unknown geometric parameters. Then, total weight per unit length of dam is chosen as goal function and the optimization problem is stablished using DE algorithm under stability, principal stress, and sliding constraints. The DE algorithm is written in MATLAB software and applied to four real gravity dams and the optimization result are compared with real size. Furthermore, optimization results of DE algorithm are compared with GA and PSOD algorithms to show the ability of DE algorithm in determining the optimal global point.

\section{Gravity Dam and Differential Evolution Based Shape Optimization}

A gravity dam was designed against proper failures. The main failure modes may be occurred due to (i) overturning/rotation about the toe; (ii) compressing or crushing; and (iii) shear sliding. The failure modes should be checked for two full and empty reservoir cases. A gravity dam design is accepted when the following stability, principal stress, and sliding constraints are satisfied.

$$
\begin{aligned}
& F O S=\frac{\sum M_{R}}{\sum M_{o}} \geq F O S_{a l l} \\
& \sigma_{c, \text { all }} \geq \sigma_{u, \max }, \sigma_{c, \text { all }} \geq \sigma_{u, \max } \\
& \tau_{c, \text { all }} \geq \tau_{u, \max }, \tau_{c, \text { all }} \geq \tau_{u, \max } \\
& F S S=\frac{\mu \Sigma F_{V}}{\Sigma F_{H}} \geq F S S_{\text {all }}
\end{aligned}
$$$$
\text { Stability Constrain }
$$

Where, $M_{R}$ and $M_{O}$ are resisting and overturning moments acting about toe. $F_{V}$ and $F_{H}$ are total vertical and horizontal forces acting on dam. $\mu$ is coefficient of friction and $\sigma_{u, \max }, \tau_{u, \max }, \sigma_{D, \max }, \tau_{D, \max }, \sigma_{c, \text { all }}$ and $\tau_{c, \text { all }}$ are principal direct and shear stresses at heel, principal direct and shear stresses at toe and allowable compressive and shear stress in concrete, respectively. Figure 1. shows a general parametric cross section of gravity dam in which the cross section are defined based on seven geometry parameters i.e., $\left(L_{1}, L_{2}, L_{3}, L_{4}, H_{1}, H_{2}, H_{3}\right)$. Indeed, these seven unknown parameter form the variables of optimization problem. By dividing Figure 1. to three main parts, all horizontal and vertical forces on gravity dam and corresponding moment about toe are presented in Table 1.

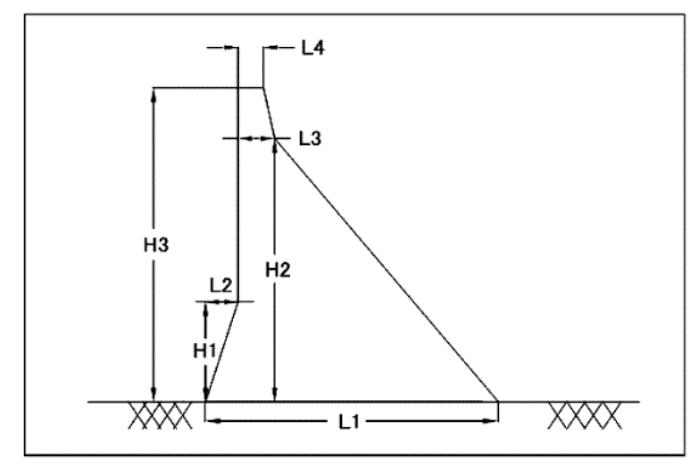

Figure 1. Parametric cross section of gravity dam 
Table 1. Horizontal and vertical forces actin on gravity dam and corresponding moments about toe

\begin{tabular}{|c|c|c|c|}
\hline No. & Load & Formula & Moment about toe \\
\hline 1 & Horizontal water pressure force & $f_{1}=\frac{1}{2} \gamma_{w} \cdot h^{2}$ & $M f_{1}=\frac{1}{3} f_{1} \cdot h$ \\
\hline 2 & Vertical water pressure force & $f_{2}=\frac{1}{2} \gamma_{w} \cdot m \cdot L_{1} \cdot h$ & $M f_{2}=f_{2} \cdot\left(L_{1}-\frac{m \cdot L_{1}}{3}\right)$ \\
\hline 3 & Weight per unit length part 1 & $W_{1}=\frac{1}{2} \gamma_{c} \cdot m \cdot L_{1} \cdot h$ & $M W_{1}=W_{1} \cdot\left(L_{1}-\frac{2}{3} m \cdot L_{1}\right)$ \\
\hline 4 & Weight per unit length part 2 & $W_{2}=\gamma_{c} \cdot L_{4} \cdot\left(h+f_{b}\right)$ & $M W_{2}=W_{2} \cdot\left(L_{1}-m \cdot L_{1}-\frac{L_{4}}{2}\right)$ \\
\hline 5 & Weight per unit length part 3 & $W_{3}=\frac{1}{2} \gamma_{c} \cdot h \cdot \frac{\left(L_{1}-m \cdot L_{1}+L_{4}\right)^{2}}{L_{1}-m \cdot L_{1}}$ & $M W_{3}=\frac{2}{3} W_{2} \cdot\left(L_{1}-m \cdot L_{1}-L_{4}\right)$ \\
\hline 6 & uplift & $u=\frac{1}{2} \gamma_{w} \cdot L_{1} \cdot h$ & $M u=\frac{2}{3} u \cdot L_{1}$ \\
\hline 7 & Static earthquake load due to W1 & $f_{e 1}=a \cdot W_{1}$ & $M f_{e 1}=\frac{1}{3} f_{e 1} \cdot h$ \\
\hline 8 & Static earthquake load due to W2 & $f_{e 2}=a \cdot W_{2}$ & $M f_{e 2}=\frac{1}{2} f_{e 2} \cdot\left(h+f_{b}\right)$ \\
\hline 9 & Static earthquake load due to W3 & $f_{e 3}=a \cdot W_{3}$ & $M f_{e 3}=\frac{1}{3} f_{e 3} \cdot h \cdot\left(\frac{L_{1}-m \cdot L_{1}-L_{4}}{L_{1}-m \cdot L_{1}}\right)$ \\
\hline 10 & $\begin{array}{l}\text { Coefficient of dynamic effect of earthquake } \\
\text { force }\end{array}$ & $c_{m}=0.73\left(\frac{90-\theta}{90}\right)$ & - \\
\hline 11 & Dynamic pressure of earthquake & $p_{e}=\gamma_{c} \cdot c_{m} \cdot a \cdot h$ & ------- \\
\hline 12 & Dynamic earthquake force & $f_{e}=0.726 p_{e} \cdot h$ & $M f_{e}=0.299 p_{e} \cdot h^{2}$ \\
\hline
\end{tabular}

Therefore, stability, principal stress, and sliding constraints Equation 1 to 3. can be rewritten as below:

$$
\begin{aligned}
& F O S=\frac{\left(M W_{1}+M W_{2}+M W_{3}+M f_{2}\right)}{\left(M f_{1}+M u+M f_{e 1}+M f_{e 2}+M f_{e 3}+M_{e}\right)} \geq F O S_{\text {all }} \\
& \sigma_{u, \max }=p_{v} \sec ^{2} \varphi-\left(p+p_{e}\right) \tan ^{2} \theta \\
& \sigma_{D, \max }=p_{v} \sec ^{2} \alpha-\left(p^{\prime}-p_{e}^{\prime}\right) \tan ^{2} \delta \\
& \tau_{u, \max }=\left[p_{v}-\left(p+p_{e}\right)\right] \tan \theta \\
& \tau_{D, \max }=\left[p_{v}-\left(p^{\prime}-p_{e}^{\prime}\right)\right] \tan \delta \\
& F S S=\frac{\mu\left(f_{2}+W_{1}+W_{2}+W_{3}-u\right)}{\left(f_{1}+f_{e 1}+f_{e 2}+f_{e 3}+f_{e}\right)} \geq F S S_{\text {all }}
\end{aligned}
$$

Where, $m$ is coefficient of crest position. The angle between vertical axis and downstream and upstream are indicated by $\delta$ and $\theta$. Also $p_{v}, p, p_{e}$, and $p_{e}^{\prime}$ imply on uplift pressure, minimum stress at heel, hydrodynamic pressure of water in absence and presence of earthquake are shown by, respectively. The optimization problem is explained at follow. Total weight per unit length of dam (sum of three parts of cross section) is considered as objective function. Gravity dam weight per unit length optimization subjected to constrain can be expressed as: 


$$
\text { Minimize } \quad W_{T}(X)=W_{1}+W_{2}+W_{3}
$$

Subject to $g_{i}(X) \leq 0$

$$
\begin{gathered}
X=\left[X_{1}, X_{2}, \ldots \ldots, X_{j}\right] \\
j=1,2, \ldots, 7
\end{gathered}
$$

In Equation 7. $x$ is the vector of design variables containing the optimization variables as $\left(L_{1}, L_{2}, L_{3}, L_{4}, H_{1}, H_{2}, H_{3}\right)$. The indices $\mathrm{i}$ and $\mathrm{j}$ are number of design variables and design constrains, respectively. Finally, six design constraints are considered as below:

$$
\begin{array}{cc}
g_{1}(X)=F_{\text {OS }}-F O S \leq 0 & g_{2}(X)=F S S_{a l l}-F S S \leq 0 \\
g_{3}(X)=\sigma_{u, \max }-\sigma_{c, a l l} \leq 0 & g_{4}(X)=\sigma_{D, \max }-\sigma_{c, a l l} \leq 0 \\
g_{5}(X)=\tau_{u, \max }-\tau_{c, a l l} \leq 0 & g_{6}(X)=\tau_{D, \max }-\tau_{c, a l l} \leq 0
\end{array}
$$

The differential evolution (DE) is a more advanced version of genetic algorithm (GA) developed by Storn and Price. The DE is an exceptional easy, very fast and powerful without encoding and decoding stages designed for optimization problems in continuous search space. It is a stochastic search algorithm and derivative-free method that search the global optimal point. The main parameters of DE approaches are NP (population size), PCR (crossover probability), and F (weight factor). In first step of DE approach, number of variables and their Lower and upper limits are determined. After considering initial values for NP, PCR, and F, a random value are attributed for $\mathrm{j}^{\text {th }}$ variable of $\mathrm{i}^{\text {th }}$ member as below:

$$
x_{i, j}=x_{j}^{10}+\operatorname{rand}_{j}[0,1]\left(x_{j}{ }^{h i}-x_{j}^{10}\right)
$$

In second step, the goal function of each member are calculated and the lowest value in the second row are stored in $f_{\min }$. The third step is mutation where for each member, three various and random members of population are selected and the following calculation are executed:

$$
y_{i, j}=x_{r 1, j}+F\left(x_{r 2, j}-x_{r 3, j}\right)
$$

In the fourth Crossover step, a $z_{j}$ population series are produced that inherits their properties from $x_{j}$ and $y_{j}$ as below:

$$
z_{j}=\left\{\begin{array}{cc}
y_{j} & r_{j} \leq P C R \quad \text { or } \quad j=j_{0} \\
x_{j} & \text { otherwise }
\end{array}\right.
$$

The last step is Selection. The main different between DE and GA approaches is selection step. In the GA, the selection of an answer as a parent is dependent on its merit value. Whilst in DE algorithm, all the answers have an equal chance to be selected. In selection step, the fittest answer is selected and passed onto the next generation. The basic structure of differential evolution (DE) algorithm is illustrated in Figure 2. 


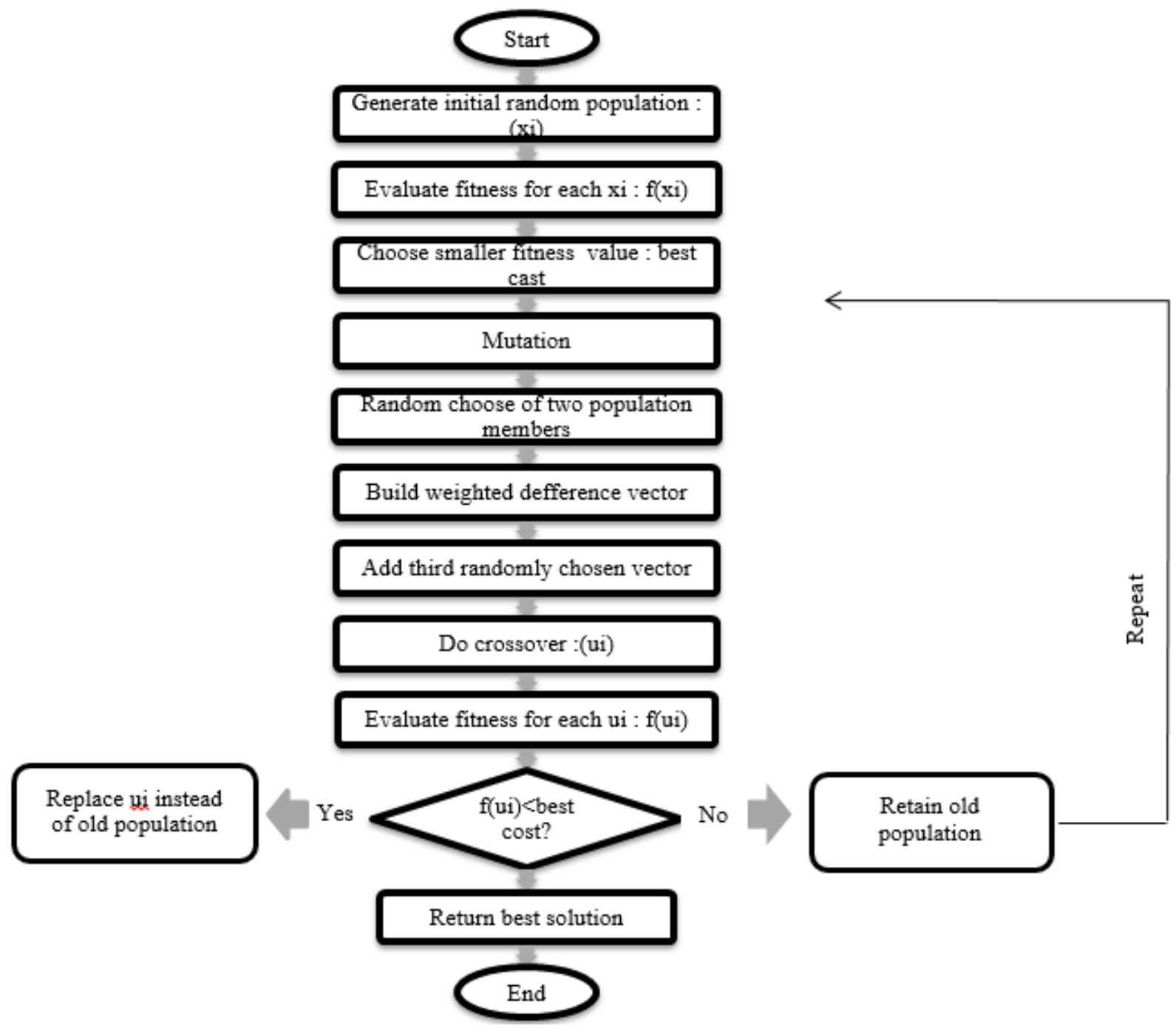

Figure 2. Basic flowchart of Differential Evolution (DE) algorithm

\section{Results and Discussion}

As mentioned, the purpose of this study is to optimize the shape of concrete gravity dam that will reduce the volume of concrete used. Hence, Differential Evolution algorithm is utilized for a pseudo static analysis based optimization of gravity dams. In this study total weight per unit length of dam is chosen as goal function and the optimization problem is established using DE algorithm under stability, principal stress, and sliding constraints. To illustrate the effectiveness of the proposed method, four concrete gravity dams including Middle Fork, Richard, Pine Flat, and Friant are selected as the real word case studies in this paper. The details of the considered dams are explained in the sequence.

The Middle Fork dam has the structural height of $32 \mathrm{~m}$ and has been constructed to control floodwater on the Middle Fork River, Colorado area in USA. The upstream floodwater and normal height level are $30 \mathrm{~m}$ and $28.96 \mathrm{~m}$ respectively. Also, the corresponding height level of downstream floodwater are $4.57 \mathrm{~m}$ and $3.5 \mathrm{~m}$. According to USRB code, drainage galleries taken into account with the distance $.05 \mathrm{~m}$ from upstream water. Second gravity dam is Richard that has the structural height of $49.68 \mathrm{~m}$ and has been constructed to control floodwater on the Savana River, Georgia USA. The upstream floodwater and normal height level are $46.63 \mathrm{~m}$ and $45.26 \mathrm{~m}$, respectively. Also, the corresponding height level of downstream floodwater are $8.83 \mathrm{~m}$ and $4.88 \mathrm{~m}$. In this dam, drainage galleries is as same as Middle Fork dam. Pine Flat is the third specimen for optimization task. This dam has the structural height of $121.92 \mathrm{~m}$ and has been constructed on the Kings River in California. The dam is constructed to control of floodwater, agriculture water supplement of downstream and production of hydropower energy. The upstream floodwater and normal height level are $116.28 \mathrm{~m}$ and $114 \mathrm{~m}$, respectively. Also, the corresponding height level of downstream floodwater are $15 \mathrm{~m}$ and $10 \mathrm{~m}$. According to USRB code, drainage galleries taken into account with the distance $0.05 \mathrm{~m}$ from upstream water.

The last selected dam is Friant. This dam has the structural height of $85.95 \mathrm{~m}$ and has been constructed on the San Joan River in California, USA. The Friant dam is also constructed to control of floodwater, agriculture water supplement of 
downstream and production of hydropower energy. The upstream floodwater and normal height level are $85.1 \mathrm{~m}$ and $84.73 \mathrm{~m}$ and the corresponding height level of downstream floodwater are $2 \mathrm{~m}$ and $1 \mathrm{~m}$.

The weights per unit length of four considered benchmark dams are $952.33,2379,13631$, and $7170 \mathrm{ton} / \mathrm{m}$, respectively. Number of initial population and maximum iteration for DE algorithm are selected as 50 and 500 for all optimization process. Convergence curve of performance index for the optimization are shown in Figure 3. According to Figure 3, the Objective function has been converged after 250, 150,310, and 350 Iterations for four mentioned dams, respectively. As seen, the DE algorithm has a high convergence accuracy and speed in addition to its ability to find the optimal global point. In maximum state, the DE algorithm has been converged in less than 350 iterations for Pine Flat Gravity Dam.

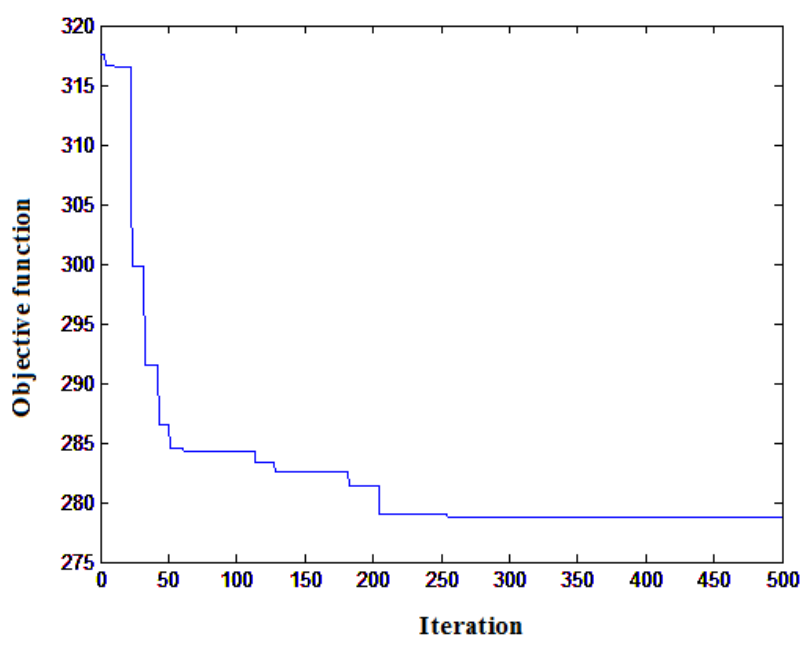

Middle Fork Gravity Dam

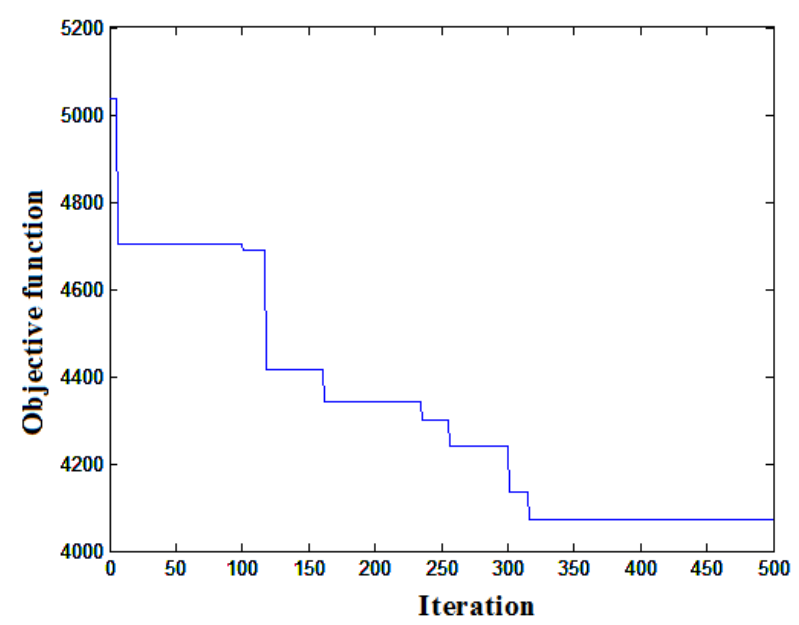

Pine Flat Gravity Dam

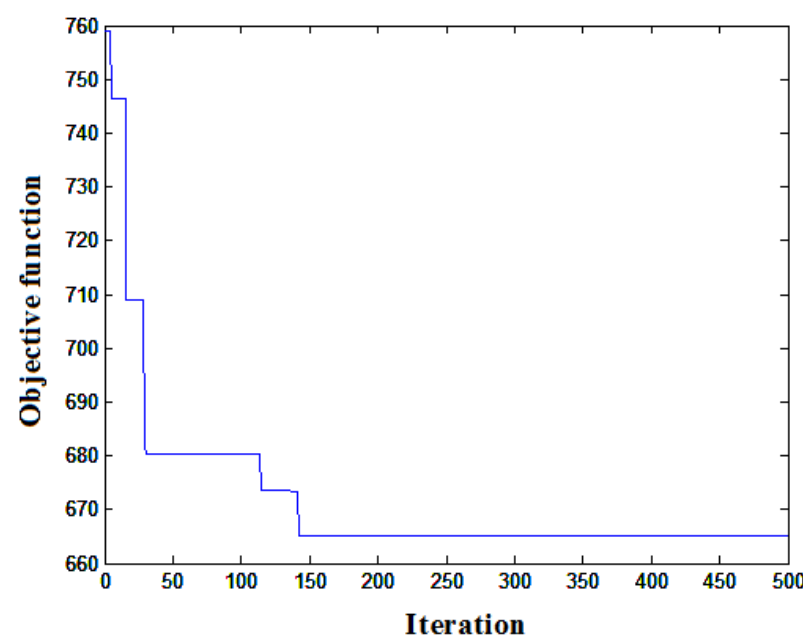

Richard Gravity Dam

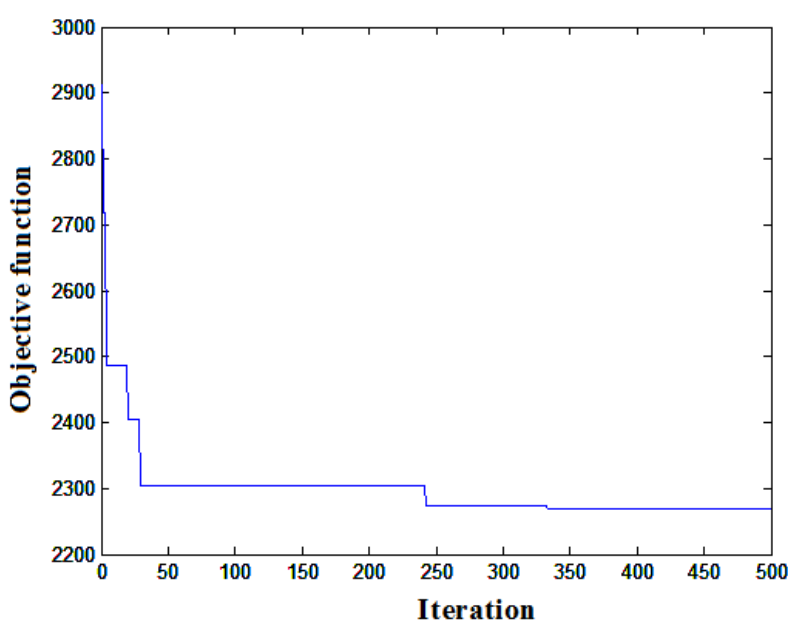

Friant Gravity Dam

Figure 3. Convergence curve of performance index in each generation for gravity dams

The optimum value of variables and corresponding objective function of benchmark dams attained by the DE algorithm and another optimization method in literature for comparison are presented in Table 2 . The results show the ability of Differentiate Evolution algorithms in optimizing the dimensions of concrete gravity dams, and thus reducing the amount of concrete used. Furthermore it can be concluded that the dam dimensions decreased rather than real dimensions. Applying the DE algorithm, we obtained $26.82 \%, 30.11 \%, 25.31 \%$, and $20.93 \%$ of reduction in weight per unit length for considered dams relative to real value, respectively. The Real and optimal cross section of four dams are presented in Figure 4 to 7. in which the reduction of the dimensions of dams is evident clearly. Finally, optimization results of DE algorithm are compared with GA and PSOD in addition to real value to study the effectiveness of the DE algorithm. The comparison shows an acceptable reduction of dimensions and weight of dams relative to GA and PSOD algorithms i.e. $3.55 \%, 5.1 \%, 19.13 \%$ and $12.14 \%$ of reduction in weight per unit length of considered dams, respectively. This considerable reduction is achieved due to the ability of DE algorithm in determining the optimal global point. 


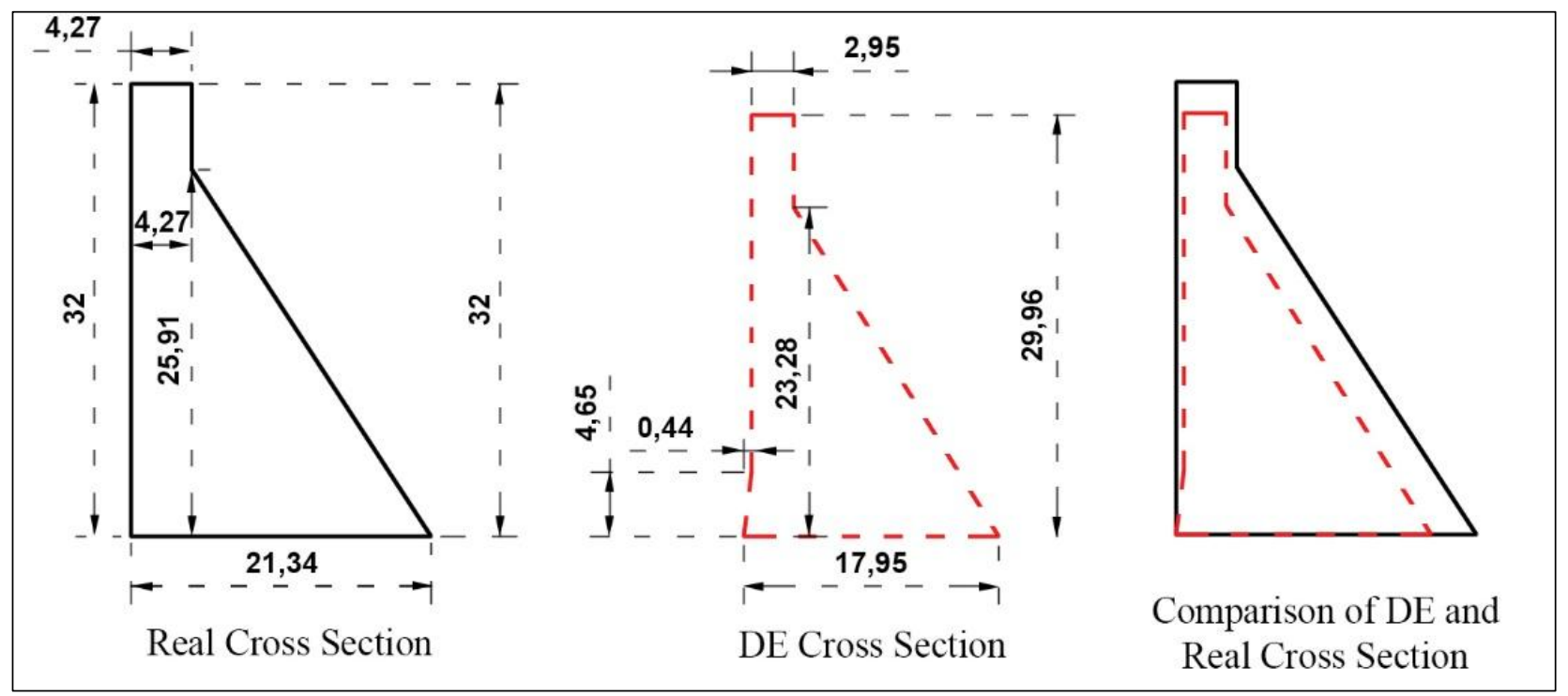

Figure 3. Real and DE based optimal cross section of Middle Fork gravity dam

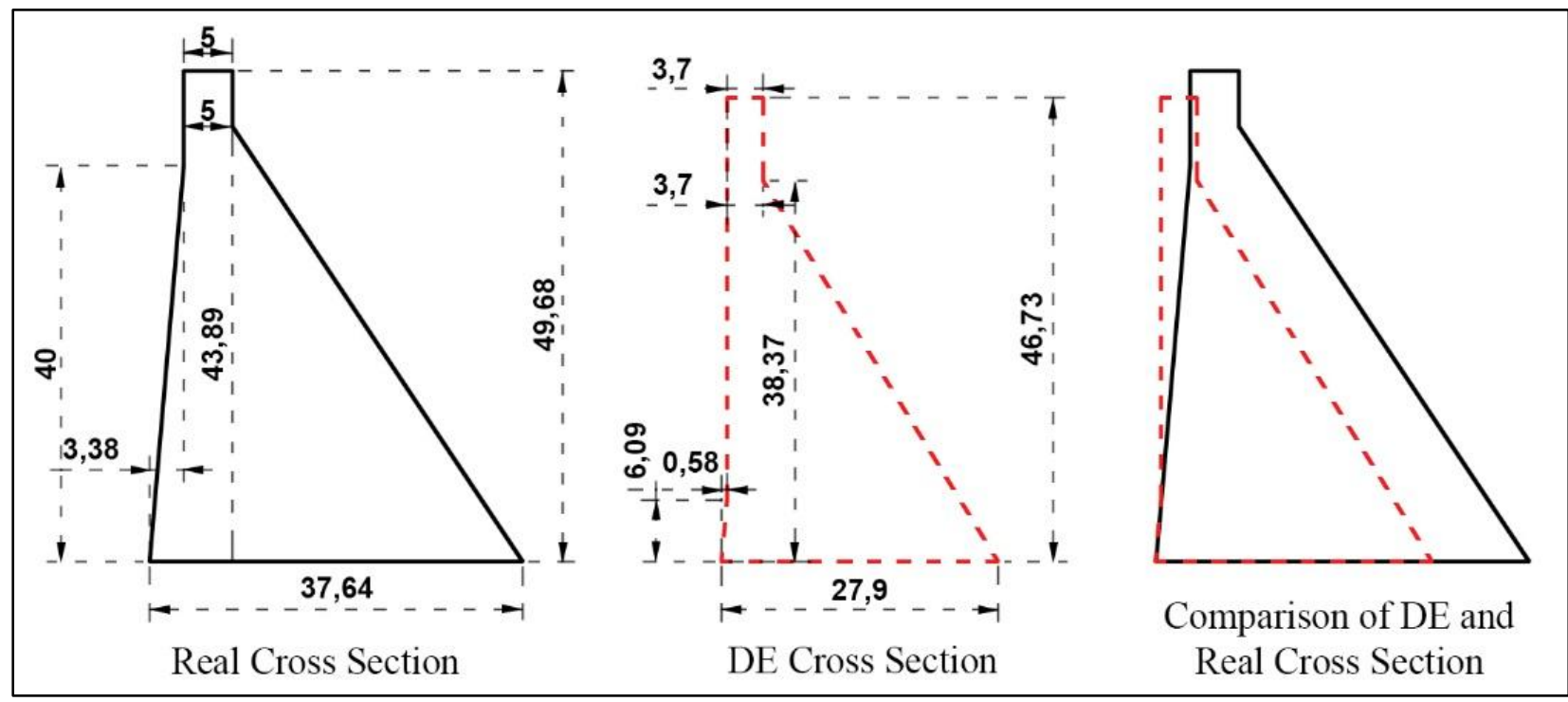

Figure 4. Real and DE based optimal cross section of Richard gravity dam

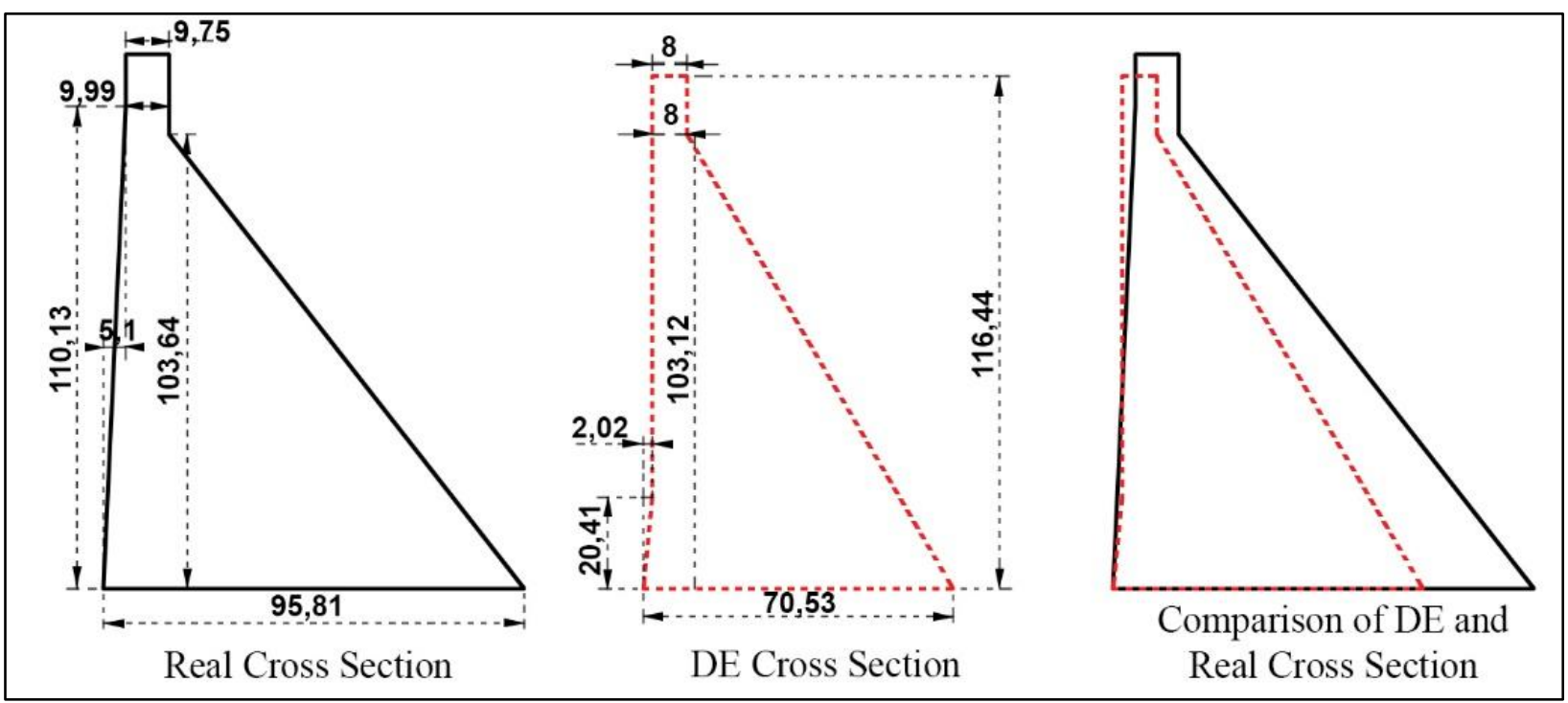

Figure 5. Real and DE based optimal cross section of Pine Flat gravity dam 


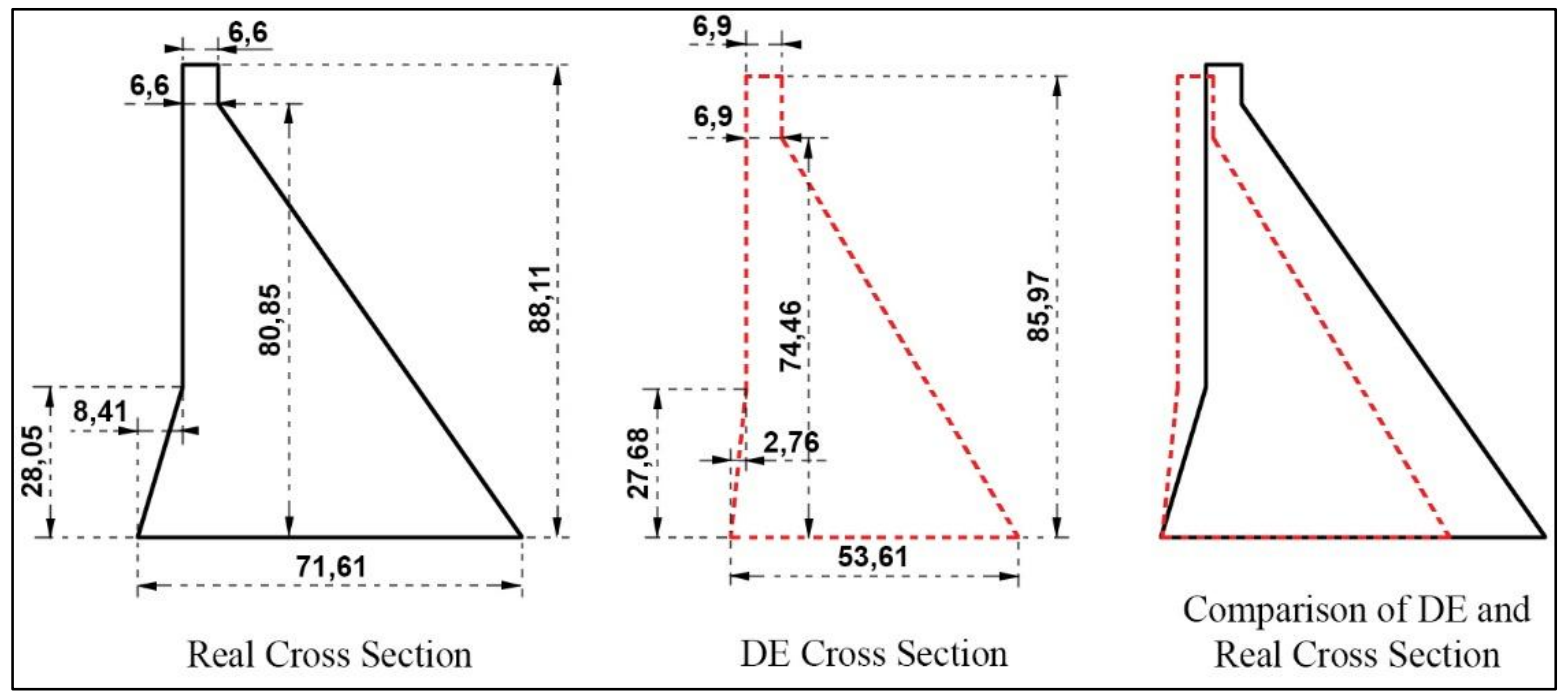

Figure 6. Real and DE based optimal cross section of Friant gravity dam

Table 2. Comparison of DE optimal cross section of Benchmark dams with real size and references [25]

\begin{tabular}{|c|c|c|c|c|c|c|c|c|}
\hline & $L_{1}(\mathrm{~m})$ & $L_{2}(\mathbf{m})$ & $L_{3}(\mathbf{m})$ & $L_{4}(\mathrm{~m})$ & $H_{1}(\mathrm{~m})$ & $\mathrm{H}_{2}(\mathrm{~m})$ & $H_{3}(\mathrm{~m})$ & $W$ (ton) \\
\hline \multicolumn{9}{|c|}{ Middle Fork Gravity Dam } \\
\hline Real Section & 21.34 & 0 & 4.27 & 4.27 & 32.00 & 25.91 & 32.00 & 952.33 \\
\hline $\mathrm{DE}$ & 17.95 & 0.44 & 2.95 & 2.95 & 4.65 & 23.28 & 29.96 & 696.95 \\
\hline GA & 19.63 & 2.06 & 6.35 & 6.35 & 30.26 & 11.93 & 32.03 & 722.59 \\
\hline PSOD & 18.51 & 1.13 & 6.00 & 6.00 & 13.48 & 16.30 & 33.32 & 720.61 \\
\hline \multicolumn{9}{|c|}{ Richard Gravity Dam } \\
\hline Real Section & 37.64 & 3.38 & 5.00 & 5.00 & 40.60 & 43.89 & 49.68 & 2379.03 \\
\hline $\mathrm{DE}$ & 27.90 & 0.58 & 3.70 & 3.70 & 6.09 & 38.37 & 46.73 & 1662.60 \\
\hline GA & 32.25 & 3.77 & 11.03 & 7.40 & 27.72 & 19.49 & 51.23 & 1751.64 \\
\hline PSOD & 31.99 & 4.01 & 6.00 & 6.00 & 27.57 & 31.51 & 52.35 & 1717.80 \\
\hline \multicolumn{9}{|c|}{ Pine Flat Gravity Dam } \\
\hline Real Section & 95.81 & 5.1 & 9.99 & 9.75 & 110.13 & 103.64 & 121.93 & 13631 \\
\hline DE Result & 70.53 & 2.02 & 8.00 & 8.00 & 20.41 & 103.12 & 116.44 & 10181 \\
\hline GA Result & 98.04 & 26.34 & 6.00 & 6.00 & 91.86 & 101.16 & 118.75 & 12589 \\
\hline \multicolumn{9}{|c|}{ Friant Gravity Dam } \\
\hline Real Section & 71.61 & 8.41 & 6.60 & 6.60 & 28.05 & 80.85 & 88.11 & 7170 \\
\hline DE Result & 53.61 & 2.76 & 6.90 & 6.90 & 27.68 & 74.46 & 85.97 & 5669.6 \\
\hline GA Result & 70.53 & 6.16 & 11.34 & 7.94 & 21.47 & 61.02 & 93.47 & 6453 \\
\hline
\end{tabular}

\section{Conclusion}

Safety and economy are two main primary goals in civil engineering problems. The economy will has special importance when a huge amount of material is used. A gravity dam is a large scale hydraulic structure providing its stability based on weight of concrete volume. Hence, optimization of dam cross-section leads to an economic and low cost structure. In this paper, a general parametric cross section of gravity dam is defined based on seven unknown horizontal and vertical geometric parameters. Weight of dam is considered as objective function and the optimization problem is solved using DE algorithm. The DE algorithm, written as a MATLAB code, are applied to four benchmark gravity dams including Middle Fork, Richard, Pine Flat, and Friant. The comparison of DE optimum solutions with real dimension of dams shows the performance of the DE algorithm. In mentioned benchmark dams, there are $26.82 \%$, $30.11 \%, 25.31 \%$, and $20.93 \%$ of reduction in weight per unit length, respectively. Furthermore, DE algorithm optimal cross section shows a remarkable reduction of objective function in comparison with GA and PSOD algorithms.

\section{References}

[1] Sahab, Mohammed Ghasem, Vassili V. Toropov, and Amir Hossein Gandomi. "A review on traditional and modern structural 
optimization: problems and techniques." Metaheuristic Applications in Structures and Infrastructures (2013): 25-47.

[2] Jang, Hyoun-Seung. "Genetic algorithm for construction space management." KSCE Journal of Civil Engineering 8, no. 4 (2004): 365-369.

[3] Marano, Giuseppe Carlo, Giuseppe Quaranta, and Giorgio Monti. "Modified genetic algorithm for the dynamic identification of structural systems using incomplete measurements." Computer - Aided Civil and Infrastructure Engineering 26, no. 2 (2011): $92-$ 110.

[4] Aydın, Zekeriya, and Yusuf Ayvaz. "Optimum topology and shape design of prestressed concrete bridge girders using a genetic algorithm." Structural and Multidisciplinary Optimization 41, no. 1 (2010): 151-162.

[5] Ashour, A. F., L. F. Alvarez, and V. V. Toropov. "Empirical modelling of shear strength of RC deep beams by genetic programming." Computers \& structures 81, no. 5 (2003): 331-338.

[6] Whigham, P. A., and P. F. Crapper. "Modelling rainfall-runoff using genetic programming." Mathematical and Computer Modelling 33, no. 6-7 (2001): 707-721.

[7] Yang, Yaowen, and Chee Kiong Soh. "Automated optimum design of structures using genetic programming." Computers \& Structures 80, no. 18 (2002): 1537-1546.

[8] Kaveh, A., and S. Talatahari. "An improved ant colony optimization for the design of planar steel frames." Engineering Structures 32, no. 3 (2010): 864-873.

[9] Reed, Martin, Aliki Yiannakou, and Roxanne Evering. "An ant colony algorithm for the multi-compartment vehicle routing problem." Applied Soft Computing 15 (2014): 169-176.

[10] Angelo, Jaqueline S., Heder S. Bernardino, and Helio JC Barbosa. "Ant colony approaches for multiobjective structural optimization problems with a cardinality constraint." Advances in Engineering Software 80 (2015): 101-115.

[11] Yang, Xin-She, and Amir Hossein Gandomi. "Bat algorithm: a novel approach for global engineering optimization." Engineering Computations 29, no. 5 (2012): 464-483.

[12] Gholizadeh, Saeed, and Amir Masoud Shahrezaei. "Optimal placement of steel plate shear walls for steel frames by bat algorithm." The Structural Design of Tall and Special Buildings 24, no. 1 (2015): 1-18.

[13] Zhou, Yongquan, Qifang Luo, Jian Xie, and Hongqing Zheng. "A hybrid bat algorithm with path relinking for the capacitated vehicle routing problem." In Metaheuristics and Optimization in Civil Engineering, pp. 255-276. Springer International Publishing, 2016.

[14] Yang, Bin, Kai-Uwe Bletzinger, Qilin Zhang, and Zhihao Zhou. "Frame structural sizing and topological optimization via a parallel implementation of a modified particle Swarm algorithm." KSCE Journal of Civil Engineering 17, no. 6 (2013): 1359-1370.

[15] Yu, Hai-yang, Shi-lian Zhang, Cong Li, and Shao-bo Wu. "Particle swarm approach for structural optimization of battleship strength deck under air blast." Journal of Shanghai Jiaotong University (Science) 19, no. 4 (2014): 481-487.

[16] Gholizadeh, Saeed, and Fayegh Fattahi. "Design optimization of tall steel buildings by a modified particle swarm algorithm." The Structural Design of Tall and Special Buildings 23, no. 4 (2014): 285-301.

[17] Erol, Osman K., and Ibrahim Eksin. "A new optimization method: big bang-big crunch." Advances in Engineering Software 37, no. 2 (2006): 106-111.

[18] Kaveh, A., and S. Talatahari. "A novel heuristic optimization method: charged system search." Acta Mechanica 213, no. 3 (2010): 267-289.

[19] Kaveh, A., and M. Khayatazad. "A new meta-heuristic method: ray optimization." Computers \& structures 112 (2012): $283-294$.

[20] Yang, X. S., "Flower pollination algorithm for global optimization. In: Unconventional Computation and Natural Computation". Lecture Notes in Computer Science, Volume 7445, (2012): 240-249.

[21] Yang, Xin-She, Gebrail Bekdaş, and Sinan Melih Nigdeli. "Review and applications of metaheuristic algorithms in civil engineering." In Metaheuristics and Optimization in Civil Engineering, pp. 1-24. Springer International Publishing, 2016.

[22] Salmasi F., "Design of gravity dam by genetic algorithm". World Academy of Science, Engineering and Technology, Volume 5, Issue 8, (2011): 755-760.

[23] Ali, Pr Dr Ahmed AM, Pr Dr Rafa HS Al-Suhaili, and Shamil AK Behaya. "A genetic algorithm optimization model for the gravity dam section under seismic excitation with reservoir-dam-foundation interactions." Amer J Eng Res 2014 3, no. 6: 143-53.

[24] Li, First A. Nannan, Second B. Zhihong Qie, Third C. Xinmiao Wu, and Fouth D. Panpan Gao. "A method based on PSO-RBF to the optimization of dam structure." In Control and Decision Conference (CCDC), 2011 Chinese, pp. 1846-1850. IEEE, 2011.

[25] A. Kaveh, P. Zakian., "Stability Based Optimum Design of Concrete Gravity Dam Using CSS, CBO and ECBO Algorithm". International Journal of Optimization in Civil Engineering, Volume 5, Number 4, (2015): 419-431.

[26] Aslani, M., A. R. Emadi, and H. Nazarpour. "Determination of appropriate values of genetic algorithm parameters in optimization of gravity dams cross section." (2013): 231-239.

[27] Khatibinia, M., H. Chiti, A. Akbarpour, and H. R. Naseri. "Shape optimization of concrete gravity dams considering dam-waterfoundation interaction and nonlinear effects." Iran University of Science \& Technology 6, no. 1 (2016): 115-134.

[28] Price, Kenneth, Rainer M. Storn, and Jouni A. Lampinen. Differential evolution: a practical approach to global optimization. Springer Science \& Business Media, 2006. 